\title{
Measuring Efficiency of Public Hospitals in Iran: A Comparative Study Using Extended Data Envelopment Analysis, 2012-2016
}

\section{Efat Mohamadi}

Tehran University of Medical Sciences

Amirhossein Takian ( $\nabla$ takian@tums.ac.ir)

Alireza Olyaee Manesh

Tehran University of Medical Sciences

\section{Reza Majdzadeh}

Tehran University of Medical Sciences

\section{Farhad Hosseinzadeh Lotfi}

Islamic Azad University

\section{Hamid Sharafi}

Islamic Azad University

Leila Hosseini Qavam Abad

Islamic Azad University

Mohammad Mehdi Kiani

Tehran University of Medical Sciences

Hasan Yusefzadeh

Urmia University of Medical Sciences

Elham Ehsani-Chimeh

Tehran University of Medical Sciences

Jalal Arabloo

Iran University of Medical Sciences

Haniye Sadat Sajadi

Tehran University of Medical Sciences

\section{Zahra Goodarzi}

Tehran University of Medical Sciences

\section{Somayeh Noori Hekmat}

Tehran University of Medical Sciences

Research 
Keywords: Data envelopment analysis (DEA), Hospital Efficiency, Productivity, Malmquist Productivity Index (MPI), Technical Efficiency, Hospital Performance

Posted Date: June 24th, 2020

DOI: https://doi.org/10.21203/rs.3.rs-36996/v1

License: (c) (1) This work is licensed under a Creative Commons Attribution 4.0 International License. Read Full License 


\section{Measuring efficiency of public hospitals in Iran: a comparative study using extended data envelopment analysis, 2012-2016}

Efat Mohamadi, Health Equity Research Center (HERC), Tehran University of Medical

Sciences (TUMS), Tehran, Iran. Efat.Mohamadi@gmail.com

\section{Amirhossein Takian (Correspond author)}

1.Health Equity Research Center (HERC), Tehran University of Medical Sciences, Tehran, Iran.

2.Department of Health Management and Economics, School of Public Health, Tehran

University of Medical Sciences, Tehran, Iran. 3.Department of Global Health and Public Policy,

School of Public Health, Tehran University of Medical Sciences, Tehran, Iran. takian@tums.ac.ir

Alireza Olyaee Manesh, 1.Health Equity ResearchCenter (HERC), TehranUniversity of

Medical Sciences, Tehran, Iran., 2.National Institute of HealthResearch, TehranUniversity of

Medical Sciences, Tehran, Iran. arolyaee@gmail.com

Reza Majdzadeh, Community Based Participatory Research Centre and Knowledge Utilization

Research Centre, Tehran University of Medical Sciences and Iran's National Institute of Health

Research, Tehran, Iran. reza.majdzadeh@gmail.com

Farhad Hosseinzadeh Lotfi, Department of Mathematics, Science and Research Branch,

Islamic Azad University, Tehran, Iran. farhad@ hosseinzadeh.ir

Hamid Sharafi , Department of Mathematics, Science and Research Branch, Islamic Azad

University, Tehran, Iran. hamid sharafi2013@yahoo.com

Leila Hosseini Qavam Abad, 1.Department of Health Care Management, Sciences and

Research Branch, Islamic Azad University, Semnan, Iran. 2. Department of Health Technology 
Assessment, Standards \& Tariffs Office, MoH, Tehran,Iran. 3. Health Equity ResearchCenter (HERC), TehranUniversity of Medical Sciences, Tehran, Iran. leila200565@yahoo.com Mohammad Mehdi Kiani, 1.Department of Health Management and Economics, School of Public Health, Tehran, Iran, 2.Health Equity Research Center (HERC), Tehran University of Medical Sciences (TUMS), Tehran, Iran. mehdi.kiyan92@gmail.com

Hasan Yusefzadeh, Assistant Professor of Health Economics, Health Management and Economics Department, School of Public Health, Urmia University of Medical Sciences, Urmia, Iran. hyusefzade2010@gmail.com

Elham Ehsani-Chimeh, National Institute for Health Research(NIHR), Tehran University of Medical Sciences, Tehran, Iran. ehsanielham@yahoo.com

Jalal Arabloo, Health Management and Economics Research Center, Iran University of Medical Sciences, Tehran, Iran. $\underline{\text { arabloo_j64@yahoo.com }}$

Haniye Sadat Sajadi, National Institute for Health Research, Tehran University of Medical Sciences, Tehran, Iran. haniehsajadi@gmail.com

Zahra Goodarzi, National Institute for Health Research, Tehran University of Medical Sciences, Tehran, Iran. zgoodarzi64@gmail.com

Somayeh Noori Hekmat, Research Center for Health Services Management, Institute for Futures Studies in Health, Kerman University of Medical Sciences, Kerman, Iran. snhekmat@gmail.com 


\section{Abstract:}

Background: Aiming to enhance quality of care and increase efficiency, public hospitals have undergone several reforms in the course of last two decades in Iran. This paper reports the result of a national research that aimed to measure the technical efficiency and productivity change of public hospitals during 2012-2016 in Iran.

Methods: We used Extended Data Envelopment Analysis (Extended-DEA) (an innovative modification to conventional DEA) to measure technical efficiency and productivity of 568 public hospitals. Nationally representative data were extracted from the official annual health reports. Data were analysed using GAMS software 24.3.

Results: The average efficiency score of all hospitals was $0.733 .10 .1 \%$ of all hospitals were efficient while $2.68 \%$ of them were under 0.2. The Malmquist Productivity Index (MPI) progressed in $49.3 \%$ of hospitals, remained constant in $2.3 \%$, while $48.2 \%$ of hospitals regressed during 2015-2016. The average of MPI was 1.07 over the period of analysis.

Conclusions: Extra efforts seem to be essential to enhance the efficient use of resources and develop appropriate policy solutions and tools. In particular, to increase the return to scale, we advocate the merger of small-size district hospitals towards establishing bigger efficient hospitals in various geographical regions across the country.

Keywords: Data envelopment analysis (DEA), Hospital Efficiency, Productivity, Malmquist Productivity Index (MPI), Technical Efficiency, Hospital Performance.

\section{Background}

Efficiency is one of the main goals of the health systems worldwide 1,2. Hospital expenditures represent around $30-50 \%$ of the total health expenditures in low-middle income countries (LMICs) 3,4, hence assessing the efficiency and productivity of hospitals is of triumph importance for any healthcare system. Further, hospital is a very complex social organization that plays a significant role in the maintenance and promotion of social health ${ }^{5}$. Pursuing their goals in providing healthcare services to citizens, education and research, the ultimate goal of a hospital is to meet the societal health needs effectively and efficiently ${ }^{6}$. Let alone, the inappropriate use of new diagnostic and treatment technologies, ageing, escalating burden of 
chronic conditions, ever-increasing demand for healthcare services, plus the consequences of health professionals' errors and their negative side effects, have all imposed heavy costs on the health systems ${ }^{7}$.

Productivity is the sum of the effectiveness (doing the right things) and the efficiency (doing the things right) of an organization. Effectiveness means achieving organizational goals, while efficiency relates to achieving the desired outputs at a lower $\cos { }^{8}$, which indicates the ratio of outputs to inputs. The goal of efficiency is to maximize benefits against the costs incurred or to minimize costs for a given benefit. Various models and methods are used to measure the performance of organizations. These include DEA, Stochastic Frontier Analysis (SFA), and efficiency indicators 9,10, among others, all of which operate based on two criteria of input (minimizing the use of inputs) and output (maximizing the output with a fixed input) ${ }^{11}$.

Many studies have been conducted on the efficiency measurement using the DEA approach in different context and countries, for example, studies of Ersoy et $\mathrm{al}^{12}$, which were among the first efforts in the field of efficiency analysis using the DEA technique, Krijia et $\mathrm{al}^{13}$, and Rhamk Rishnan ${ }^{14}$, Ghaderi et $\mathrm{al}^{15}$, Ardekani et $\mathrm{al}^{16}$, and Azad et $\mathrm{al}^{17}$, have used the frontier data analysis method to evaluate the efficiency of hospitals.

In study of Pérez-Romero ${ }^{18}$, concluded 230 NHS hospitals, the average rate of overall technical efficiency (OTE) was 0.736 in 2012. Also, Dong et al, conducted a systematic review and result showed statistical significances were found in indicators such as number of Decision Making Units (DMUs), percentage of allocative efficiency studies, ratio of studies with multiple years, number of studies with monetary indicators in input and output sets, etc ${ }^{19}$. Another systematic review conducted by Pelone et al(reviewed 39 DEA applications in PC) to understand how methodological frameworks impact results and influence the information provided to decision makers. Studies were combined using qualitative narrative synthesis. This paper reports data for each efficiency analysis on the: 1) evaluation context; 2) model specifications; 3) application of methods to test the robustness of findings; 4) presentation of results ${ }^{20}$. A systematic review conducted with another approach to search articles applying combined DEA and SFA in order to facilitate a common comprehension about the adequacy of these methods, defining any differences in healthcare efficiency estimation and the reasons that are behind this ${ }^{21}$.

Several studies have used DEA to assess the efficiency of hospitals, including in Iran ${ }^{22}$.

These studies have alluded to, although partially, the inefficiency of public hospitals, between 0.584 and 0.998 in Iran ${ }^{17}$. For instance, a comparison between the average length of stay and average occupancy rate indices in hospitals in Iran with other LMICs reveals the inappropriate utilization of existing resources ${ }^{23,24}$. Nevertheless, all studies used a single method for calculating the efficiency, and as a result, failed to provide a comprehensive picture of the 
hospitals' efficiency nationwide. No previous research has determined the current status of efficiency within all public hospitals in Iran ${ }^{25}$.

In the course of last two decades, public hospitals in Iran have undergone several reforms to enhance quality of care ${ }^{26}$ and increase efficiency, i.e. decentralization, accreditation, and since 2014, improving hospitals' productivity through health transformation plan (HTP) ${ }^{27}$. Our team conducted a national research to measure the efficiency of the entire health system in Iran. This paper reports the findings of a study that aimed to measure the efficiency of all public hospitals during 2012-2016 in Iran.

\section{Methods}

\section{Setting}

Both private and public sectors contribute to providing hospital care in Iran. All hospitals are regulated under the supervision of the ministry of health and medical education (MoHME). In 2016, there were 921 active hospitals in the country, 80\% of them were governmental and 20\% were nongovernmental hospitals (Appendix 1), scattered across 31 provinces in Iran. In this research, we included governmental public hospitals, affiliated to the MoHME, and divided them into two categories: general and specialized hospitals. General hospitals were divided into three sub-categories: medical-non-educational, medical-educational, and medical- educationalresearch centers. Specialized hospitals were divided into eight sub-categories: Orthopedic, Accidents and Burns, Pediatric, Ophthalmology, Psychiatry, Gynecology and Obstetrics, Cardiology, Cancer and Oncology (This classification has been made by the MoHME). The average bed occupancy in public hospitals was 73\% in $2014{ }^{28}$.

\section{Study design}

This was a quantitative and descriptive- analytical study. Our sample included all governmental public hospitals affiliated to the MoHME in Iran. We extracted data from secondary databases linked to the MoHME's health information system (HIS). We measured the efficiency score, the MPI and provided the benchmark for each of the indicators. First, we conducted a literature review and used the classic DEA method to measure efficiency. However, the initial results did not make sense for the research team. This was because the units that used only minimal inputs were efficient, while the health output had not been adjusted for both quality and equity aspects simultaneously. In other words, the reality of resource distribution, their case mix, and other contextual factors that may affect hospitals efficiency, were not taken into consideration in DEA conventional method. To overcome this challenge, we, in collaboration with a scholarly team in applied mathematics began a modification process, so-called extended-DEA to balance and rationalize the results. We will explain the three consecutive steps below: 


\section{Step 1: definition of input-output indicators}

We conducted a qualitative analysis, i.e. literature review and collecting experts' opinions, to identify the input and output indicators. First, a scoping review of related studies identified a list of related indicators to the objectives of our research ${ }^{29}$. Second, we examined the existence of data associated with each indicator and the reliability of the data source, according to which, many indicators were excluded. Finally, the included indicators were reviewed and approved by an expert panel, comprising of the research team plus selected key informants in the field of heath management, policy and economics (Appendix 2).

\section{Step 2: data collection and cleaning}

We used a checklist for data collection that was designed based on the input and output variables and the years studied. An Excel sheet was used to enter the data, acquired from the hospitals and workforce information database of the MoHME, for all public hospitals, as “Decision Making Units (DMUs)".

We then cleaned up the data to ensure the existence and accuracy of all data for each indicator per each DMU throughout all years of the study period. Irregular data was compared with other sources to ensure data integrity. We include all Iranian public hospitals, but due to limited number of input and output indicators; we exclude the DMUs without data for one indicator in one particular year (or years). Data collection and cleaning lasted six months. Following the opinions of selected key informants, we classified hospitals based on their specialty, teaching and non-teaching, as well as their performance indicators. To compare heterogeneous hospitals included in our study, we used the "level of specialty" variable, which let us classify similar hospitals in certain designated groups in a meaningful manner and conduct a meaningful fair comparison ${ }^{30}$ ( Appendix 2).

\section{Step 3: data analysis and modelling}

Each indicator was weighed and given a value using the standards set of the MoHME as well as the enjoying the views of an external advisory board. The more important an indicator was classified, the more influence it had on the efficiency score (Appendix 2).

DEA is a mathematically-based technique to determine the relative efficiency of a congruent DMUs.

Initially, in the DMU community, a point is determined and fixed as a benchmark for the DMU under evaluation, on the basis of alleviating the policies defined by the management. Subsequently, relative efficiency of the DMU under assessment is calculated on the basis of benchmarking, which ranges between 0 and 1 . The efficiency of a DMU under evaluation is signified by equating to 1 , whereas, if this value is less than 1, it denotes the inefficiency of the DMU under investigation. Therefore, higher efficiency could be a sign of the DMU's better performance. In this article, we considered each hospital as a DMU, while hospitals were 
categorized into various specialty groups and EDEA models were independently implemented to each categorization.

We spouse to each hospital uses 4 inputs to create 7 outputs. we use the following symbols to show the values of inputs and outputs of the hospital $j(j=1, \ldots, n)$.

$\mathrm{x}_{\mathrm{ij}}$ : Value of ith input of hospital $\mathrm{j}, \mathrm{i}=1, . ., 4, \mathrm{j}=1, \ldots, \mathrm{n}$.

$\mathrm{y}_{\mathrm{rj}}$ : Value of rth output of hospital $\mathrm{j}, \mathrm{r}=1, \ldots, 7, \mathrm{j}=1, \ldots, \mathrm{n}$.

As described above, we determined the inputs and outputs for each hospital for modelling as follows:

\begin{tabular}{|l|l|l|l|}
\hline \multicolumn{1}{|c|}{ Inputs } & \multicolumn{1}{|c|}{ Symbols } & \multicolumn{1}{c|}{ Outputs } & \multicolumn{1}{c|}{ Symbols } \\
\hline n. Physician & $x_{1 j}$ & n. Inpatient & (D) $y_{1 j}$ \\
\hline n. Nurse & $x_{2 j}$ & n. Outpatient & (D) $y_{2 j}$ \\
\hline n. Other staff & $x_{3 j}$ & n. Surgical operation & (D) $y_{3 j}$ \\
\hline & $x_{4 j}$ Hospital bed & Degree of accreditation & (D) $y_{4 j}$ \\
& & Average length of stay & (U.D) $y_{5 j}$ \\
\hline & & & \\
& & Bed Occupancy & (D) $y_{6 j}$ \\
\hline
\end{tabular}

Symbols D and U.D are desirable and undesirable respectively. In other words, increase of desirable outputs is considered by management which improves productivity. However, the undesirable outputs are not considered by manager which have adverse effect on productivity. Since the fifth output (Average length of stay) is an undesirable output. We make the following changes to make it a desirable output.

$\mathrm{y}_{5 \mathrm{j} \text { new }}=1 / \mathrm{y}_{5 \mathrm{j}}$ previous

As we mentioned in method, given the definitions of each the input and output, the following constraints are taken for them based on the opinions of experts.
$\mathrm{v}_{1} \geq 1.3 \mathrm{v}_{2}$,
$\mathrm{u}_{7} \geq \mathrm{u}_{1}$,
$\mathrm{u}_{6} \geq \mathrm{u}_{3}$
$\mathrm{v}_{2} \geq 1.3 \mathrm{v}_{3}$,
$\mathrm{u}_{2} \geq \mathrm{u}_{1}$,
$\mathrm{u}_{3} \geq 1.5 \mathrm{u}_{4}$
$\mathrm{v}_{2} \geq 3.9 \mathrm{v}_{4}$,
$\mathrm{u}_{3} \geq \mathrm{u}_{5}$,

Relationships (2) show relative weight of indicators. For example, the importance of the seventh output is at least equal to the first output, and the importance of the first input to the second 
input is at least 1.3. Since the design of this research requires a restriction, the modeling is done in envelopment form. Therefore, constraints (2) appear in trade-off in the envelopment form with symbols $\alpha$ and $\beta$. Also, the variables $\gamma$ and $\mu$ are correspond of this trade-off in envelopment form.

On the other hand, the sixth output is expressed as a "percentage", so its value must always be between $[0,100]$. Therefore, the following constraints are considered in modelling.

$0 \leq \sum_{\mathrm{j}=1}^{315} \lambda_{\mathrm{j}} \mathrm{y}_{6 \mathrm{j}}+\sum_{\mathrm{j}=1}^{5} \gamma_{\mathrm{j}} \beta_{6 \mathrm{j}} \leq 100$

The number of bed days is also dependent on the number of bed, which is why the following model constraints are considered in modelling.

$\sum_{\mathrm{j}=1}^{315} \lambda_{\mathrm{j}} \mathrm{y}_{7 \mathrm{j}}+\sum_{\mathrm{j}=1}^{5} \gamma_{\mathrm{j}} \beta_{7 \mathrm{j}} \leq 365 *\left(\sum_{\mathrm{j}=1}^{315} \lambda_{\mathrm{j}} \mathrm{x}_{4 \mathrm{j}}+\sum_{\mathrm{j}=1}^{3} \mu_{\mathrm{j}} \alpha_{4 \mathrm{j}}\right)$

According to the above description, the radial model in the envelopment form, taking into according to the trade-off and limitations on the template, will be as follows:

Min $\theta$

$$
\begin{aligned}
& \text { s.t. } \quad \sum_{j=1}^{n} \lambda_{j} x_{1 j}+\sum_{j=1}^{3} \mu_{j} \alpha_{4 j} \leq \theta x_{i p}, \quad i=1, \ldots, 4 \text {, } \\
& \sum_{\mathrm{j}=1}^{\mathrm{n}} \lambda_{\mathrm{j}} \mathrm{y}_{\mathrm{rj}}+\sum_{\mathrm{j}=1}^{5} \gamma_{\mathrm{j}} \beta_{\mathrm{rj}} \geq \mathrm{y}_{\mathrm{rp}}, \quad \mathrm{r}=1, \ldots, 7, \\
& \sum_{\mathrm{j}=1}^{\mathrm{n}} \lambda_{\mathrm{j}} \mathrm{y}_{6 \mathrm{j}}+\sum_{\mathrm{j}=1}^{5} \gamma_{\mathrm{j}} \beta_{6 \mathrm{j}} \leq 100 \\
& \sum_{j=1}^{n} \lambda_{j} y_{6 j}+\sum_{j=1}^{5} \gamma_{j} \beta_{6 j} \geq 0 \\
& \sum_{j=1}^{n} \lambda_{j} y_{7 j}+\sum_{j=1}^{5} \gamma_{j} \beta_{7 j} \leq 365 *\left(\sum_{j=1}^{n} \lambda_{j} x_{4 j}+\sum_{j=1}^{3} \mu_{j} \alpha_{4 j}\right) \\
& \lambda_{\mathrm{j}} \geq 0, \quad \mathrm{j}=1, \ldots, \mathrm{n}, \\
& \gamma_{\mathrm{j}} \geq 0, \quad \mathrm{j}=1, \ldots, 5 \text {, } \\
& \mu_{\mathrm{j}} \geq 0, \quad \mathrm{j}=1,2,3 \text {. }
\end{aligned}
$$

The final model to calculate the relative

(a) efficiency of hospital $\mathrm{p}$ has come to hand by

(b) solving the model hereunder:

The optimal value of the objective function of the model (5) can be denoted as a relative efficiency of hospital p. It is obvious that, if the optimal value of the objective function of model (5) is equivalent to 1 , then hospital p (efficient). Similarly, if the optimal value of the objective function of model (5) is less than 1, then the hospital p can be called as being inefficient, so its coordinates of benchmark will be as the following: 
$\operatorname{Benchmark}=\left(\sum_{\mathrm{j}=1}^{\mathrm{n}} \lambda_{\mathrm{j}}^{*} \mathrm{x}_{1 \mathrm{j}}+\sum_{\mathrm{j}=1}^{\mathrm{n}^{\prime}} \mu^{*}{ }_{\mathrm{j}} \alpha_{4 \mathrm{j}}, \sum_{\mathrm{j}=1}^{\mathrm{n}} \lambda^{*}{ }_{\mathrm{j}} \mathrm{y}_{\mathrm{rj}}+\sum_{\mathrm{j}=1}^{\mathrm{n}^{\prime \prime}} \gamma_{\mathrm{j}}{ }_{\mathrm{j}} \beta_{\mathrm{rj}}\right)$

In order to calculate the progressive and unprogressive aspects of each of the hospitals on the basis of efficiency or performance, the Malmquist Productivity Index (MPI) has been computed. This index is derived from the comparison of efficiency changes to technological modifications 31, according to which, we divided hospitals into three groups:

- Hospitals showing progress during (if MPI $>1$ );

- Hospitals showing regression (if $\mathrm{MPI}<1$ ); and

Hospitals whose performance remained constant during their period of study (if MPI=1).

The notation $\left(x_{p}^{2}, y_{p}^{2}\right),\left(x_{p}^{1}, y_{p}^{1}\right)$ for allocating to $D M U_{p}(p=1, \ldots, n)$ is used in periods 1 and 2 respectively. Two factors are effective in measuring productivity:

${ }^{(i)}$ Catch-up Effect $(\Delta \mathrm{E})$ : A degree that indicates the improvement or deterioration in efficiency and is calculated as follows:

$\Delta \mathrm{E}=\frac{\delta_{2}^{2}}{\delta_{1}^{1}}$

Where $\delta_{t+1}^{t}$ is efficiency at time $t+1$ to real time $t$. In other words, the Catch-up Effect is the efficiency in the second to first periods.

(ii ) Frontier-shift Effect5: Calculates the boundaries of performance between the two periods and calculates the following.

$\Delta \mathrm{T}=\sqrt{\frac{\delta_{1}^{2} \times \delta_{2}^{2}}{\delta_{1}^{1} \times \delta_{2}^{1}}}$

The Malmquist Index is the ratio of efficiency changes and efficiency boundary changes that calculated as follows.

$$
\left(\frac{\Delta \mathrm{E}}{\Delta \mathrm{T}}\right)=\left[\frac{\delta_{2}^{1} \times \delta_{2}^{2}}{\delta_{1}^{1} \times \delta_{1}^{2}}\right]^{\frac{1}{2}} \quad M I=
$$

For example, we solve the following model for $\delta_{2}^{1}$. 


$$
\begin{aligned}
& \delta_{2}^{1}=\operatorname{Min} \quad \theta \\
& \text { s.t. } \quad \sum_{j=1}^{n} \lambda_{j} x_{1 j}^{1}+\sum_{j=1}^{3} \mu_{j} \alpha_{4 j} \leq \theta x_{i p}^{2}, \quad i=1, \ldots, 4 \text {, } \\
& \sum_{j=1}^{n} \lambda_{j} y_{r j}^{1}+\sum_{j=1}^{5} \gamma_{j} \beta_{r j} \geq y_{r p}^{2}, \quad r=1, \ldots, 7, \\
& \sum_{\mathrm{j}=1}^{\mathrm{n}} \lambda_{\mathrm{j}} \mathrm{y}_{6 \mathrm{j}}^{1}+\sum_{\mathrm{j}=1}^{5} \gamma_{\mathrm{j}} \beta_{6 \mathrm{j}} \leq 100 \\
& \sum_{\mathrm{j}=1}^{\mathrm{n}} \lambda_{\mathrm{j}} \mathrm{y}_{6 \mathrm{j}}^{1}+\sum_{\mathrm{j}=1}^{5} \gamma_{\mathrm{j}} \beta_{6 \mathrm{j}} \geq 0 \\
& \sum_{j=1}^{n} \lambda_{j} y_{7 j}^{1}+\sum_{j=1}^{5} \gamma_{j} \beta_{7 j} \leq 365 *\left(\sum_{j=1}^{315} \lambda_{j} x_{4 j}^{1}+\sum_{j=1}^{3} \mu_{j} \alpha_{4 j}\right) \\
& \lambda_{\mathrm{j}} \geq 0, \quad \mathrm{j}=1, \ldots, 315, \\
& \gamma_{\mathrm{j}} \geq 0, \quad \mathrm{j}=1, \ldots, 5, \\
& \mu_{\mathrm{j}} \geq 0, \quad \mathrm{j}=1,2,3 \text {. }
\end{aligned}
$$

\section{Results}

We analyzed 568 hospitals within 2 categories and 11 subcategories. Initially, we began our study based on conventional DEA to measure the efficiency of hospitals. The primary results were difficult to interpret in the context of the Iranian healthcare system. Therefore, we started to categorized hospitals based on their specialty, the mix case, whether they are research oriented and/or train residents and fellows. To determine this criteria, we convened an expert meeting of some pioneers, including chancellors of medical universities, officials of the MOHME, some hospital managers and academics. Appendix 3 is a summary of categorized hospitals.

The descriptive statistics of inputs, outputs and explanatory variables are shown in Tables 1 and 2. We summarized the efficiency score and MPI of hospitals in Tables 3 and 4 . Tables 5 and 6 show the efficiency score and MPI of the general and specialized hospitals during 2012-2016 in Iran. Finally, Table 7 presents the total inputs that need to be reduced and the outputs that need to be promoted for 2015 .

Psychiatry Hospitals are kind of specialized hospitals, but we report all of the data and results of this group of hospitals separately to prevent the impact of their indicators on results. The 
standards and constrains of performance indicators in these hospitals are different from other specialized hospitals; for example, we set " $X<3.5$ " for "Average length of stay" indicator, while the Average length of stay in Psychiatry Hospitals is more than Twenty days.

Table 1. Descriptive statistics of inputs and explanatory variables

\begin{tabular}{|c|c|c|c|c|c|c|c|c|c|c|c|c|c|c|c|c|}
\hline \multirow{2}{*}{$\begin{array}{c}\text { Input } \\
\text { variables }\end{array}$} & \multirow{2}{*}{$\begin{array}{l}\text { Summary } \\
\text { statistics }\end{array}$} & \multicolumn{5}{|c|}{ General Hospitals } & \multicolumn{5}{|c|}{ Specialized Hospitals } & \multicolumn{5}{|c|}{ Psychiatry Hospitals } \\
\hline & & 2012 & 2013 & 2014 & 2015 & 2016 & 2012 & 2013 & 2014 & 2015 & 2016 & 2012 & 2013 & 2014 & 2015 & 2016 \\
\hline \multirow{6}{*}{$\begin{array}{l}\text { n. } \\
\text { Physician }\end{array}$} & Sum & 14917 & 17263 & 19052 & 18990 & 20670 & 2180 & 2513 & 2772 & 2777 & 2997 & 335 & 393 & 440 & 435 & 466 \\
\hline & Max & 286 & 332 & 365 & 362 & 389 & 129 & 149 & 164 & 163 & 175 & 32 & 37 & 40 & 40 & 43 \\
\hline & Min & 2 & 3 & 2 & 2 & 2 & 2 & 2 & 2 & 2 & 2 & 4 & 4 & 5 & 5 & 5 \\
\hline & Mean & 33.6 & 38.9 & 42.4 & 41.6 & 44.4 & 25.6 & 29.2 & 32.2 & 31.9 & 34.4 & 12.9 & 14.6 & 15.7 & 15.5 & 16.6 \\
\hline & SD & 32.3 & 37.4 & 41.1 & 40.4 & 43.3 & 22.1 & 25.5 & 28.1 & 27.9 & 29.8 & 8.2 & 9.6 & 10.3 & 10.3 & 10.9 \\
\hline & Median & 24 & 27 & 29 & 29 & 31 & 18 & 20.5 & 23 & 22 & 24 & 10 & 11 & 11.5 & 11.5 & 12.5 \\
\hline \multirow[t]{6}{*}{ n. Nurse } & Sum & 68327 & 74579 & 74743 & 74203 & 91307 & 10611 & 11579 & 11561 & 11793 & 14495 & 2239 & 2499 & 2553 & 2527 & 3055 \\
\hline & Max & 1114 & 1215 & 1212 & 1200 & 1464 & 712 & 777 & 775 & 767 & 936 & 202 & 221 & 220 & 218 & 266 \\
\hline & Min & 15 & 17 & 23 & 21 & 25 & 14 & 15 & 15 & 15 & 18 & 18 & 19 & 19 & 19 & 23 \\
\hline & Mean & 153.9 & 168.0 & 166.5 & 162.0 & 195.5 & 124.8 & 134.6 & 134.4 & 137.1 & 166.6 & 86.1 & 92.6 & 91.2 & 90.3 & 109.1 \\
\hline & SD & 142.0 & 154.3 & 153.5 & 150.6 & 183.3 & 100.4 & 109.2 & 108.9 & $\begin{array}{ll}14.9 \\
\end{array}$ & 139.5 & 52.5 & 56.6 & 55.8 & 55.3 & 66.3 \\
\hline & Medic & 113 & 124 & 123 & 120.5 & 147 & 105 & 112.5 & 112.5 & 111.5 & 130 & 68 & 70 & 68 & 67.5 & 82 \\
\hline \multirow{6}{*}{$\begin{array}{l}\text { n. Other } \\
\text { staff }\end{array}$} & Sum & 77982 & 78055 & 83868 & 82291 & 90456 & 14325 & 14410 & 15375 & 15110 & 16685 & 3124 & 3175 & 3509 & 3438 & 3707 \\
\hline & Max & 1945 & 1947 & 2085 & 2043 & 2227 & 886 & 886 & 949 & 930 & 1014 & 584 & 585 & 626 & 614 & 627 \\
\hline & Min & 13 & 18 & 19 & 21 & 26 & 18 & 18 & 20 & 19 & 21 & 17 & 17 & 18 & 17 & 19 \\
\hline & Mean & 174.8 & 175.0 & 186.4 & 179.7 & 194.1 & 168.5 & 167.6 & 178.8 & 175.7 & 191.8 & 120.2 & 117.6 & 125.3 & 122.8 & 132.4 \\
\hline & SD & 178.2 & 178.4 & 190.5 & 185.2 & 200.1 & 138.5 & 138.5 & 147.5 & 145.0 & 160.9 & 115.5 & 114.3 & 120.1 & 117.8 & 122.0 \\
\hline & Median & 129.5 & 129.5 & 138 & 133 & 144 & 139 & 139 & 149 & 146 & 159 & 89.5 & 87 & 96 & 94 & 102.5 \\
\hline \multirow{6}{*}{$\begin{array}{l}\text { n. } \\
\text { Hospital } \\
\text { bed }\end{array}$} & Sum & 61078 & 61517 & 62418 & 63787 & 67145 & 12825 & 12917 & 12218 & 12585 & 12858 & 7374 & 7432 & 6362 & 6208 & 6209 \\
\hline & Max & 973 & 973 & 909 & 871 & 860 & 2159 & 2159 & 1165 & 1177 & 1177 & 2159 & 2159 & 1165 & 1177 & 1177 \\
\hline & Min & 23 & 23 & 27 & 27 & 27 & 16 & 18 & 18 & 18 & 18 & 47 & 47 & 19 & 32 & 32 \\
\hline & Mean & 137.6 & 138.6 & 138.7 & 139.0 & 143.8 & 150.9 & 150.2 & 142.1 & 146.3 & 147.8 & 283.6 & 275.3 & 227.2 & 221.7 & 221.8 \\
\hline & SD & 127.9 & 128.8 & 128.5 & 128.7 & 132.8 & 243.2 & 241.6 & 151.7 & 153.2 & 153.7 & 425.6 & 419.2 & 259.1 & 239.9 & 240.6 \\
\hline & Median & 108.5 & 109 & 106 & 107 & 110 & 104 & 105.5 & 107.5 & 112 & 114 & 177.5 & 175 & 170 & 176 & 176 \\
\hline
\end{tabular}


Table 2. Descriptive statistics of outputs and explanatory variables.

\begin{tabular}{|c|c|c|c|c|c|c|c|c|c|c|c|c|c|c|c|c|}
\hline \multirow{2}{*}{$\begin{array}{l}\text { Output } \\
\text { variables }\end{array}$} & \multirow{2}{*}{$\begin{array}{l}\text { Summary } \\
\text { statistics }\end{array}$} & \multicolumn{5}{|c|}{ General Hospitals $(\mathrm{N}=467)$} & \multicolumn{5}{|c|}{ Specialized Hospitals ( $N=73$ ) } & \multicolumn{5}{|c|}{ Psychiatry Hospitals ( $\mathrm{N}=28$ ) } \\
\hline & & 2012 & 2013 & 2014 & 2015 & 2016 & 2012 & 2013 & 2014 & 2015 & 2016 & 2012 & 2013 & 2014 & 2015 & 2016 \\
\hline \multirow[t]{6}{*}{ n. Inpatient } & Sum & 5287795 & 5523056 & 5762557 & 6230901 & 6483827 & 841920 & 880945 & 905318 & 959929 & 982771 & 80644 & 101308 & 81890 & 92606 & 94523 \\
\hline & Max & 90157 & 90157 & 86479 & 93868 & 95706 & 48618 & 50932 & 54988 & 55379 & 57858 & 16218 & 16218 & 15105 & 14870 & 13191 \\
\hline & Min & 22 & 22 & 31 & 146 & 93 & 256 & 325 & 305 & 249 & 332 & 256 & 325 & 156 & 249 & 332 \\
\hline & Mean & 11909.4 & 12439.3 & 12834.2 & 13634.4 & 13884.0 & 9904.9 & 10243.5 & 10527.0 & 11162.0 & 11296.2 & 3101.7 & 3752.1 & 2924.6 & 3307.4 & 3375.8 \\
\hline & SD & 11425.5 & 11340.1 & 11992.9 & 12295.6 & 12876.1 & 9377.4 & 9245.3 & 10394.7 & 10177.8 & 10519.7 & 3236.8 & 3596.9 & 3028.2 & 3138.5 & 3055.4 \\
\hline & Median & 10,003 & 10,608 & 10,651 & 11,863 & 11,840 & 8,020 & 8,049 & 7,769 & 8,678 & 7,920 & 1,883 & 2,406 & 1,780 & 2,654 & 2,599 \\
\hline \multirow[t]{6}{*}{ n. Outpatient } & Sum & $\begin{array}{c}26347902 \\
1\end{array}$ & $\begin{array}{c}32632047 \\
7\end{array}$ & $\begin{array}{c}34480578 \\
2\end{array}$ & $\begin{array}{c}59247796 \\
1\end{array}$ & $\begin{array}{c}65959251 \\
5\end{array}$ & 40019821 & 50725069 & $\begin{array}{c}5349973 \\
5\end{array}$ & 70178688 & 71158752 & $\begin{array}{c}7547671 . \\
5\end{array}$ & $\begin{array}{c}9635074 . \\
3\end{array}$ & 11997864 & 15266615 & 39101261 \\
\hline & Max & 6217523 & 6771325 & 7443500 & 13571550 & 13542100 & 2633856 & 3292320 & 3465600 & 4891850 & 4884500 & $\begin{array}{c}2653097 . \\
3\end{array}$ & $\begin{array}{c}3316371 . \\
6\end{array}$ & $\begin{array}{c}3490917 . \\
5\end{array}$ & 5090860 & 19635125 \\
\hline & Min & 1462 & 1828 & 1924 & 2028 & 2258 & 2953 & 3691 & 3885 & 3726 & 3104 & 47 & 47 & 46 & 47 & 48 \\
\hline & Mean & 593421 & 734956 & 766235 & 1296451 & 1415435 & 470821 & 589826 & 622090 & 816031 & 817917 & 290295 & 356855 & 428495 & 545236 & 1396474 \\
\hline & SD & 862507.8 & 1061558.8 & 1119376.9 & 1652515.1 & 1712257.8 & 568590.3 & 714386.9 & 753874.1 & $\begin{array}{c}1041391 . \\
2\end{array}$ & $\begin{array}{c}1032358 . \\
6\end{array}$ & 558246.9 & 685137.9 & 760983.2 & $\begin{array}{c}1032480 . \\
7\end{array}$ & $\begin{array}{c}3889545 . \\
4\end{array}$ \\
\hline & Median & 271320 & 337402 & 347855 & 794440 & 917730 & 298802 & 340148 & 358050 & 425880 & 442220 & 71,091 & 88,265 & 113,180 & 148,650 & 228,700 \\
\hline \multirow{6}{*}{$\begin{array}{l}\text { n. Surgical } \\
\text { operation }\end{array}$} & Sum & 2376015 & 2407909 & 2652601 & $\begin{array}{l}2838129 \\
\end{array}$ & 2990767 & 361483 & 377386 & 433591 & 443593 & 467554 & 21662 & 26172 & 30434 & 30457 & 30931 \\
\hline & Max & 40674 & 36093 & 38878 & 42006 & 44318 & 55438 & 66930 & 74015 & 83072 & 88607 & 6125 & 6846 & 6912 & 6874 & 6752 \\
\hline & Min & 6091 & 6457 & 7602 & 7873 & 8806 & 149 & 165 & 231 & 242 & 228 & 0 & 0 & 0 & 0 & 0 \\
\hline & Mean & 5099 & 5167 & 5620 & 5925 & 6116 & 4253 & 4388 & 5042 & 5158 & 5374 & 833 & 969 & 1087 & 1088 & 1105 \\
\hline & SD & 5330 & 5296 & 5676 & 5936 & 6160 & 6781 & 7898 & 8885 & 9788 & 10389 & 1610 & 1887 & 1911 & 1878 & 1916 \\
\hline & Median & 3,609 & 3,524 & 3,951 & 4,117 & 4,241 & 2,912 & 2,585 & 2,734 & 2,760 & 2,894 & 1,600 & 2,115 & 2,638 & 2,468 & 2,742 \\
\hline \multirow{5}{*}{$\begin{array}{l}\text { Average } \\
\text { length of } \\
\text { stay }\end{array}$} & Max & 7.6 & 7.4 & 7.4 & 6.4 & 10.2 & 104.2 & 102.1 & 101.1 & 118.6 & 83.9 & 339.7 & 333.0 & 329.7 & 389.4 & 117.2 \\
\hline & Min & 1.0302 & 1.01 & 1 & 1 & 1 & $\begin{array}{c}0.985901 \\
4\end{array}$ & 1 & 0.957 & 1 & 1 & $\begin{array}{c}2.450845 \\
8\end{array}$ & 2.40279 & 2.379 & 2.279 & 2.388 \\
\hline & Mean & 2.6 & 2.5 & 2.5 & 2.5 & 2.6 & 8.2 & 8.1 & 8.0 & 7.8 & 6.8 & 39.8 & 38.1 & 36.7 & 37.0 & 23.9 \\
\hline & SD & 0.9 & 0.9 & 0.9 & 0.9 & 1.0 & 17.2 & 16.8 & 16.6 & 17.7 & 12.1 & 66.2 & 63.9 & 62.3 & 73.9 & 24.8 \\
\hline & Median & 2.4 & 2.3 & 2.3 & 2.4 & 2.4 & 2.9 & 2.9 & 2.8 & 2.8 & 2.9 & 18.8 & 18.2 & 17.9 & 16.2 & 16.7 \\
\hline \multirow{5}{*}{$\begin{array}{l}\text { Bed } \\
\text { occupation } \\
(\%)\end{array}$} & Max & 100.0 & 100.0 & 100.0 & 100.0 & 100.0 & 100.0 & 100.0 & 100.0 & 100.0 & 100.0 & 100.0 & 100.0 & 100.0 & 100.0 & 100.0 \\
\hline & Min & 4.7 & 4.3 & 5 & 9.3 & 9.8 & 10.04404 & 10.24902 & 10.566 & 13.998 & 12.484 & $\begin{array}{c}31.14355 \\
7\end{array}$ & 31.77914 & 32.762 & 54.974 & 62.846 \\
\hline & Mean & 59.4 & 60.6 & 62.0 & 68.3 & 67.5 & 68.6 & 69.8 & 72.0 & 73.2 & 73.5 & 75.1 & 75.8 & 77.0 & 80.7 & 84.6 \\
\hline & SD & 19.8 & 20.2 & 21.1 & 20.9 & 21.5 & 19.1 & 19.5 & 20.0 & 20.0 & 18.5 & 13.4 & 14.0 & 15.5 & 10.8 & 9.8 \\
\hline & Median & 63.48 & 64.77 & 66.63 & 72.70 & 72.16 & 72.21 & 73.68 & 75.96 & 78.10 & 78.97 & 77.21 & 78.52 & 80.36 & 81.88 & 86.74 \\
\hline \multirow[t]{6}{*}{$\begin{array}{l}\begin{array}{l}\text { Number } \\
\text { bed days }\end{array} \\
\end{array}$} & Sum & 14795982 & 15574706 & 16075248 & 17692832 & 19072542 & $\begin{array}{c}3059691 . \\
8 \\
\end{array}$ & $\begin{array}{c}3236509 \\
8 \\
\end{array}$ & 3336591 & 3510362 & $\begin{array}{c}3362968 . \\
1 \\
\end{array}$ & $\begin{array}{c}1687675 . \\
6 \\
\end{array}$ & $\begin{array}{c}1792351 . \\
5 \\
\end{array}$ & 1849818 & 1833137 & 1671906 \\
\hline & Max & 226937 & 238881 & 246269 & 263241 & 342302 & 303974 & 319973 & 329869 & 325735 & 220594 & 303974 & 319973 & 329869 & 325735 & 220594 \\
\hline & Min & 29 & 30 & 31 & 129 & 16 & 1066 & 1122 & 1157 & 1411 & 1367 & 5510 & 5800 & 2033 & 6242 & 8990 \\
\hline & Mean & 33324 & 35078 & 35723 & 38800 & 40928 & 35996 & 37634 & 38798 & 40818 & 39104 & 64911 & 66383 & 66065 & 65469 & 59711 \\
\hline & SD & 35919 & 37809 & 38904 & 41257 & 45171 & 43402 & 45480 & 46887 & 46897 & 38132 & 71400 & 74387 & 76293 & 69647 & 49952 \\
\hline & Median & 23834 & 25088 & 25609 & 28579 & 29422 & 26600 & 27823 & 28684 & 29100 & 29527 & 47645 & 47244 & 47597 & 53144 & 55920 \\
\hline \multirow{5}{*}{$\begin{array}{l}\text { Degree of } \\
\text { accreditation }\end{array}$} & Max & 4 & 4 & 4 & 4 & 4 & 4 & 4 & 4 & 4 & 3 & 4 & 4 & 4 & 4 & 3 \\
\hline & Min & 1 & 1 & 1 & 1 & 1 & 1 & 1 & 1 & 1 & 1 & 1 & 1 & 1 & 1 & 1 \\
\hline & Mean & 3 & 3 & 3 & 3 & 3 & 3 & 3 & 3 & 3 & 3 & 3 & 3 & 3 & 3 & 3 \\
\hline & SD & 0.6 & 0.6 & 0.7 & 0.7 & 0.6 & 0.5 & 0.5 & 0.6 & 0.5 & 0.4 & 0.6 & 0.6 & 0.7 & 0.7 & 0.5 \\
\hline & Median & 3 & 3 & 3 & 3 & 3 & 3 & 3 & 3 & 3 & 3 & 3 & 3 & 3 & 3 & 3 \\
\hline
\end{tabular}


3 Tables 3 and 4 present overall results of the efficiency scores and MPI. The average 4 efficiency score of all hospitals was $0.733 .10 .11 \%$ of all hospitals had a score of 1 and $52.68 \%$ of them had efficiency scores below 0.2 . MPI had also progressed in $49.3 \%$ of 6 hospitals, $2.3 \%$ of them did not change and $48.2 \%$ of them had regressed in 20157 2016. The mean of MPI was 1.07 over the analysis years.

9 Table 3. Overall efficiency results 2012-16.

\begin{tabular}{|c|c|c|c|c|c|c|c|}
\hline \multicolumn{2}{|c|}{ Summary statistics } & 2012 & 2013 & 2014 & 2015 & 2016 & Mean \\
\hline \multicolumn{2}{|l|}{$\mathbf{N}$} & 542 & 543 & 549 & 557 & 568 & 551 \\
\hline \multicolumn{2}{|c|}{ Mean } & 0.732 & 0.731 & 0.718 & 0.735 & 0.748 & 0.733 \\
\hline \multicolumn{2}{|c|}{ Max } & 1 & 1 & 1 & 1 & 1 & 1 \\
\hline \multicolumn{2}{|c|}{ Min } & 0.158 & 0.158 & 0.159 & 0.158 & 0.159 & 0.159 \\
\hline \multicolumn{2}{|l|}{ SD } & 0.166 & 0.166 & 0.163 & 0.172 & 0.159 & 0.150 \\
\hline \multicolumn{2}{|c|}{ Median } & 0.709 & 0.691 & 0.684 & 0.729 & 0.729 & 0.712 \\
\hline \multicolumn{2}{|c|}{ Efficient (N) } & 56 & 56 & 54 & 57 & 56 & 56 \\
\hline \multicolumn{2}{|c|}{ Efficient (\%) } & 10.3 & 10.3 & 9.8 & 10.2 & 9.9 & 10.1 \\
\hline \multicolumn{2}{|c|}{ Inefficient(N) } & 486 & 487 & 495 & 500 & 512 & 496 \\
\hline \multicolumn{2}{|c|}{ Inefficient (\%) } & 89.7 & 89.7 & 90.2 & 89.8 & 90.1 & 89.9 \\
\hline \multirow{11}{*}{ 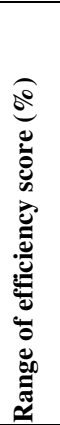 } & $E<0.1$ & 0 & 0 & 0 & 0 & 0 & 0 \\
\hline & $0.1 \leq \mathrm{E}<0.2$ & 3.14 & 2.76 & 3.10 & 1.44 & 2.99 & 2.68 \\
\hline & $0.2 \leq \mathrm{E}<0.3$ & 18.45 & 22.47 & 15.85 & 14.00 & 20.42 & 18.24 \\
\hline & $0.3 \leq \mathrm{E}<0.4$ & 23.99 & 20.44 & 24.04 & 23.70 & 20.42 & 22.52 \\
\hline & $0.4 \leq \mathrm{E}<0.5$ & 23.06 & 23.39 & 21.86 & 24.06 & 23.42 & 23.16 \\
\hline & $0.5 \leq \mathrm{E}<0.6$ & 6.64 & 5.89 & 9.65 & 10.59 & 7.22 & 8.00 \\
\hline & $0.6 \leq \mathrm{E}<0.7$ & 11.62 & 11.79 & 12.20 & 12.03 & 11.80 & 11.89 \\
\hline & $0.7 \leq \mathrm{E}<0.8$ & 2.03 & 2.21 & 2.55 & 3.23 & 3.35 & 2.67 \\
\hline & $0.8 \leq \mathrm{E}<0.9$ & 0.74 & 0.74 & 0.91 & 0.72 & 0.53 & 0.73 \\
\hline & $0.9 \leq \mathrm{E}<0.99$ & 0 & 0 & 0 & 0 & 0 & 0.00 \\
\hline & $E=1$ & 10.33 & 10.31 & 9.84 & 10.23 & 9.86 & 10.11 \\
\hline
\end{tabular}

11 Table 4. Overall MPI results, 2012-16.

\begin{tabular}{|c|c|c|c|c|c|}
\hline \multicolumn{2}{|c|}{ Summary statistics } & $2012-2013$ & 2013-2014 & 2014-2015 & 2015-2016 \\
\hline \multicolumn{2}{|c|}{ Mean } & 1.041 & 1.009 & 1.189 & 1.043 \\
\hline \multicolumn{2}{|c|}{ Max } & 1.269 & 1.82 & 8.541 & 6 \\
\hline \multicolumn{2}{|c|}{ Min } & 0.619 & 0.593 & 0.266 & 0.256 \\
\hline \multicolumn{2}{|l|}{ SD } & 0.053 & 0.057 & 0.335 & 0.290 \\
\hline \multicolumn{2}{|c|}{ Median } & 1.049 & 1.017 & 1.086 & 0.992 \\
\hline \multirow{6}{*}{ 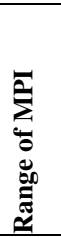 } & MPI>1 (N) & 491 & 374 & 450 & 275 \\
\hline & MPI>1 (\%) & 90.59 & 68.75 & 82.12 & 49.37 \\
\hline & MPI=1 (N) & 9 & 11 & 6 & 13 \\
\hline & MPI=1 (\%) & 1.66 & 2.02 & 1.09 & 2.33 \\
\hline & MPI<1 (N) & 42 & 159 & 92 & 269 \\
\hline & MPI $<1(\%)$ & 7.75 & 29.23 & 16.79 & 48.29 \\
\hline
\end{tabular}

12 The average efficiency in specialized hospitals i.e. Cancer and Oncology, Orthopedic 13 and Ophthalmology was higher than other hospitals. Medical and non-educational 14 hospitals had the lowest efficiency scores (Figure 1). We indicate extended data 15 envelopment analysis models are independently implemented to each categorization. Hence, 
the hospitals in the different categorizations face the different technologies, and the efficiencies of the hospitals cannot be compared among categorizations. The purpose of this figure is to show the average efficiency at a glance.

\section{Figure 1. Average efficiency score by hospital type, 2012-16.}

Table 5 shows that, on average, 7 out of 315 general hospitals had the efficiency scores of above 0.8 , with the mean efficiency score of 0.357 over the analysis years. The lowest efficiency score was in $2016(\mathrm{M}=0.157, \mathrm{SD}=0.15)$. The variation range $(\mathrm{R})$ of the scores in this group was high $(\mathrm{R}=0.841)$. The MPI improved in most general hospitals (Mean=220); the highest and the lowest improvement was during 2014-2015 (MPI = 8.54) and 2015-2016 (MPI = 0.256), respectively.

The mean efficiency score in teaching hospitals was less than $0.5(\mathrm{M}=0.488)$, the lowest score observed in 2014 ( $\mathrm{Min}=0.333$ ). The range of efficiency scores in these hospitals was 0.645 . In the study years, on average, 7 teaching hospitals had a score of above 0.8. The MPI mean score in teaching hospitals indicates a slight improvement in hospital efficiency score (MPI=1.02). On average, 94 of 130 teaching hospitals had an MPI of more than 1. The lowest (0.561) and the highest (1.710) progress in this index was during 2014-2015 and 2015-2016, respectively.

The average efficiency scores in teaching and research hospitals was 0.760 over the study years, while the lowest score observed in 2013 -2014 (Min=0.576). On average, 8 out of 22 hospitals had a score of above 0.8 in this group. The range of efficiency scores was 0.405 in these hospitals. The highest MPI score was in 2014-2015 ( $R=4.460)$. On average, the lowest improvement was 0.768 in this index. In addition, 16 out of 22 hospitals had efficiency improvement over the analysis years.

The average efficiency score of orthopedic hospitals was 0.899 over the analysis years. The lowest efficiency score in these hospitals was in 2015 (Min=0.588); with the lowest average in 2015 (0.814) and the highest average in 2014 (0.923). Three hospitals were mostly efficient $(\mathrm{E}>0.8)$ in this group. The MPI in orthopedic hospitals showed progress in 2012 and 2013. Efficiency scores were progressed in 50\% of hospitals during 2013-2016 ( $\mathrm{N}=397)$. The highest progress rate (1.96) was observed in 2015-2016.

Among nine accidents \& burn specialist hospitals, three hospitals had a score of above 0.8 . The range of scores was 0.425 in this group, which was lower than that of general hospitals. Minimum score and standard deviation were 0.574 and 0.16 , respectively, in this group. 
The average efficiency score in 13 specialized pediatric hospitals was 0.796 ( $\mathrm{SD}=0.15$, $\mathrm{R}=0.353$ ), five of which operated efficiently ( $\mathrm{E}>0.8$ ) with the average efficiency score of 0.646. On average, the MPI showed progress in nine pediatric hospitals during the analysis years, with the highest improvement in 2014-2015 (MPI=2.429). The average efficiency improvement in these hospitals was 1.08, while the average efficiency score was $0.863(\mathrm{R}=0.21, \mathrm{SD}=0.18)$.

The efficiency scores showed progress in most Ophthalmology hospitals, with the maximum improvement in 2015-2016 (MPI = 2.832). The average MPI was 1.2 in this group.

The average efficiency score was $0.733(R=0.395)$ in psychiatric hospitals, with the minimum efficiency score of $0.604(\mathrm{SD}=0.11)$. Seven out of the total of 28 psychiatric hospitals had a score of above 0.8. On average, the efficiency score in 21 psychiatric specialized hospitals showed progress (MPI> 1). The highest progress was in 20152016 (MPI=3.98), while 2014-2015 showed the biggest efficiency regression $(\mathrm{MPI}=0.84)$ in this group.

The average efficiency score in gynecology and obstetrics specialized hospitals was $0.740(\mathrm{R}=0.192)$. Out of 25 hospitals in this group, nine showed the efficiency scores higher than 0.8 , while the minimum efficiency score was $0.579(\mathrm{SD}=0.14, \mathrm{R}=0.42$ ) during the study years. On average, four out of 25 hospitals showed progress in their scores (MPI> 1). The maximum MPI progress was 1.439 during 2014-2015.

The average efficiency score in nine specialized Cardiology hospitals was 0.769 $(\mathrm{SD}=0.16)$. On average, three hospitals were efficient $(\mathrm{E}>0.8)$ and the range of efficiency scores was 0.376. The MPI progressed in all Cardiology hospitals 20122013, and regressed 2013-2016. The highest MPI score was observed 2015-2016 (MPI=1.12) in this group.

The average efficiency score was $0.866(\mathrm{R}=0.337)$ in cancer and oncology hospitals, with the minimum efficiency score of $0.663(\mathrm{SD}=0.12)$. Moreover, the MPI progressed in cancer and oncology hospitals 2012-2014 (MPI=1.05) and regressed during 20142016 (MPI=0.97).

Table 5. Efficiency score and MPI of general hospitals, and their frequency distribution, 2012-2016. 


\begin{tabular}{|c|c|c|c|c|c|c|c|c|c|c|c|}
\hline \multirow{2}{*}{$\begin{array}{l}\text { Specialty } \\
\text { of } \\
\text { hospitals }\end{array}$} & \multirow{2}{*}{$\begin{array}{l}\text { Summary } \\
\text { statistics }\end{array}$} & \multicolumn{6}{|c|}{ Efficiency score } & \multicolumn{4}{|l|}{ MPI } \\
\hline & & 2012 & 2013 & 2014 & 2015 & 2016 & Mean & 2012-2013 & 2013-2014 & 2014-2015 & $2015-2016$ \\
\hline \multirow{5}{*}{ 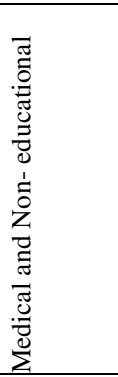 } & Min & 0.158 & 0.158 & 0.159 & 0.158 & 0.159 & 0.159 & 0.619 & 0.593 & 0.266 & 0.256 \\
\hline & Mean & 0.356 & 0.354 & 0.368 & 0.386 & 0.349 & 0.357 & 1.047 & 1.005 & 1.265 & 1.063 \\
\hline & SD & 0.161 & 0.177 & 0.168 & 0.158 & 0.147 & 0.138 & 0.058 & 0.091 & 0.524 & 0.435 \\
\hline & $\mathrm{CV}$ & 0.454 & 0.501 & 0.455 & 0.410 & 0.423 & 0.385 & 0.056 & 0.091 & 0.414 & 0.409 \\
\hline & $\begin{array}{l}\text { No. of } \\
E>0.8 / \\
M P I>1\end{array}$ & 12 & 16 & 13 & 11 & 9 & 7 & 268 & 196 & 258 & 157 \\
\hline \multirow{4}{*}{ 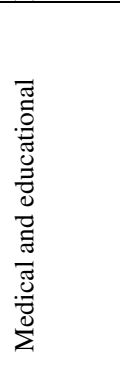 } & SD & 0.133 & 0.105 & 0.132 & 0.130 & 0.139 & 0.101 & 0.034 & 0.058 & 0.119 & 0.121 \\
\hline & Median & 0.438 & 0.444 & 0.448 & 0.465 & 0.461 & 0.452 & 1.020 & 1.031 & 1.058 & 1 \\
\hline & $\mathrm{CV}$ & 0.278 & 0.224 & 0.271 & 0.261 & 0.274 & 0.208 & 0.033 & 0.058 & 0.111 & 0.120 \\
\hline & $\begin{array}{l}\text { No. of } \\
E>0.8 / \\
M P I>1\end{array}$ & 7 & 4 & 7 & 7 & 8 & 4 & 112 & 100 & 104 & 62 \\
\hline \multirow{2}{*}{$\vec{\Xi}$} & Max & 1 & 1 & 1 & 1 & 1 & 1 & 1.177 & 1.130 & 4.460 & 1.274 \\
\hline & Min & 0.593 & 0.577 & 0.577 & 0.621 & 0.604 & 0.594 & 0.997 & 0.924 & 0.768 & 0.873 \\
\hline 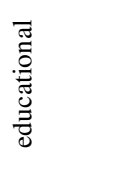 & Mean & 0.777 & 0.753 & 0.745 & 0.765 & 0.761 & 0.760 & 1.055 & 1.016 & 1.272 & 1.014 \\
\hline
\end{tabular}

92

Table 6. Efficiency score and MPI of specialized hospitals, and their frequency distribution, 20122016.

\begin{tabular}{|c|c|c|c|c|c|c|c|c|c|c|c|}
\hline \multirow{2}{*}{$\begin{array}{l}\text { Special } \\
\text { ty of } \\
\text { hospita } \\
\text { ls }\end{array}$} & \multirow{2}{*}{$\begin{array}{l}\text { Summary } \\
\text { statistics }\end{array}$} & \multicolumn{6}{|c|}{ Efficiency score } & \multicolumn{4}{|l|}{ MPI } \\
\hline & & 2012 & 2013 & 2014 & 2015 & 2016 & Mean & $\begin{array}{l}2012- \\
2013\end{array}$ & 2013-2014 & 2014-2015 & $\begin{array}{l}2015- \\
2016\end{array}$ \\
\hline \multirow{5}{*}{$\begin{array}{l}: 0 \\
0 \\
00 \\
0 \\
0 \\
0 \\
0\end{array}$} & Min & 0.685 & $\begin{array}{c}0.68 \\
6\end{array}$ & 0.691 & $\begin{array}{c}0.58 \\
8\end{array}$ & $\begin{array}{c}0.66 \\
9\end{array}$ & 0.680 & 1.011 & 0.849 & 0.452 & 0.886 \\
\hline & Mean & 0.921 & $\begin{array}{c}0.92 \\
1\end{array}$ & 0.923 & $\begin{array}{c}0.81 \\
4\end{array}$ & $\begin{array}{c}0.91 \\
7\end{array}$ & 0.899 & 1.080 & 0.972 & 0.908 & 1.216 \\
\hline & SD & 0.158 & $\begin{array}{c}0.15 \\
7\end{array}$ & 0.154 & $\begin{array}{c}0.21 \\
7\end{array}$ & $\begin{array}{c}0.16 \\
5\end{array}$ & 0.151 & 0.061 & 0.088 & 0.316 & 0.507 \\
\hline & Median & 1 & 1 & 1 & $\begin{array}{c}0.83 \\
3\end{array}$ & 1 & 0.958 & 1.082 & 0.999 & 1.006 & 1.0055 \\
\hline & $\mathrm{CV}$ & 0.171 & $\begin{array}{c}0.17 \\
0\end{array}$ & 0.167 & $\begin{array}{c}0.26 \\
7\end{array}$ & $\begin{array}{c}0.18 \\
0\end{array}$ & 0.168 & 0.056 & 0.090 & 0.348 & 0.417 \\
\hline \multirow{5}{*}{ 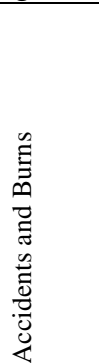 } & Min & 0.569 & $\begin{array}{c}0.56 \\
9\end{array}$ & 0.556 & $\begin{array}{c}0.56 \\
5\end{array}$ & $\begin{array}{c}0.60 \\
7\end{array}$ & 0.575 & 0.985 & 0.955 & 0.702 & 0.838 \\
\hline & Mean & 0.774 & $\begin{array}{c}0.77 \\
1\end{array}$ & 0.727 & $\begin{array}{c}0.82 \\
6\end{array}$ & $\begin{array}{c}0.83 \\
5\end{array}$ & 0.787 & 1.039 & 1.006 & 1.091 & 0.964 \\
\hline & SD & 0.191 & $\begin{array}{c}0.19 \\
2 \\
\end{array}$ & 0.175 & $\begin{array}{c}0.20 \\
0\end{array}$ & $\begin{array}{c}0.19 \\
0\end{array}$ & 0.165 & 0.044 & 0.037 & 0.271 & 0.083 \\
\hline & Median & 0.680 & $\begin{array}{c}0.66 \\
9 \\
\end{array}$ & 0.648 & $\begin{array}{c}0.90 \\
7 \\
\end{array}$ & $\begin{array}{c}0.83 \\
2 \\
\end{array}$ & 0.799 & 1.039 & 1.011 & 1.166 & 0.949 \\
\hline & $\mathrm{CV}$ & 0.246 & $\begin{array}{c}0.24 \\
9\end{array}$ & 0.241 & $\begin{array}{c}0.24 \\
2\end{array}$ & $\begin{array}{c}0.22 \\
7\end{array}$ & 0.210 & 0.042 & 0.037 & 0.248 & 0.086 \\
\hline
\end{tabular}




\begin{tabular}{|c|c|c|c|c|c|c|c|c|c|c|c|}
\hline & $\begin{array}{l}\text { No. } \\
\text { E>0.8/ } \\
\text { MPI }>1\end{array}$ & 3 & 3 & 2 & 5 & 5 & 3 & 6 & 4 & 5 & 3 \\
\hline \multirow[b]{7}{*}{ 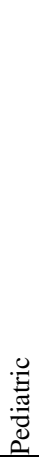 } & Max & 1 & 1 & 1 & 1 & 1 & 1 & 1.148 & 1.038 & 2.429 & 1.527 \\
\hline & Min & 0.593 & $\begin{array}{c}0.59 \\
3 \\
\end{array}$ & 0.577 & $\begin{array}{c}0.63 \\
3 \\
\end{array}$ & $\begin{array}{c}0.67 \\
8 \\
\end{array}$ & 0.646 & 0.946 & 0.953 & 0.955 & 0.796 \\
\hline & Mean & 0.794 & $\begin{array}{c}0.79 \\
5\end{array}$ & 0.766 & $\begin{array}{c}0.78 \\
0\end{array}$ & $\begin{array}{c}0.84 \\
6\end{array}$ & 0.796 & 1.043 & 1.010 & 1.253 & 1.018 \\
\hline & SD & 0.172 & $\begin{array}{c}0.17 \\
1\end{array}$ & 0.168 & $\begin{array}{c}0.15 \\
8\end{array}$ & $\begin{array}{c}0.15 \\
2\end{array}$ & 0.156 & 0.056 & 0.029 & 0.373 & 0.183 \\
\hline & Median & 0.688 & $\begin{array}{c}0.68 \\
9\end{array}$ & 0.673 & $\begin{array}{c}0.71 \\
4\end{array}$ & $\begin{array}{c}0.80 \\
0\end{array}$ & 0.694 & 1.034 & 1.018 & 1.168 & 0.971 \\
\hline & $\mathrm{CV}$ & 0.216 & $\begin{array}{c}0.21 \\
5\end{array}$ & 0.219 & $\begin{array}{c}0.20 \\
3\end{array}$ & $\begin{array}{c}0.17 \\
9\end{array}$ & 0.196 & 0.054 & 0.028 & 0.298 & 0.180 \\
\hline & $\begin{array}{ll}\text { No. } & \text { of } \\
\text { E }>0.8 & \\
\text { /MPI }>1 & \\
\end{array}$ & 5 & 5 & 4 & 4 & 7 & 5 & 11 & 9 & 11 & 5 \\
\hline \multirow{7}{*}{ 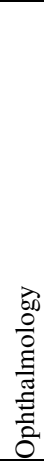 } & Max & 1 & 1 & 1 & 1 & 1 & 1 & 1.203 & 1.043 & 2.49 & 2.832 \\
\hline & Min & 0.611 & $\begin{array}{c}0.61 \\
2 \\
\end{array}$ & 0.611 & $\begin{array}{c}0.60 \\
9 \\
\end{array}$ & $\begin{array}{c}0.62 \\
8 \\
\end{array}$ & 0.619 & 0.993 & 0.991 & 0.891 & 0.642 \\
\hline & Mean & 0.873 & $\begin{array}{c}0.87 \\
3 \\
\end{array}$ & 0.873 & $\begin{array}{c}0.87 \\
7 \\
\end{array}$ & $\begin{array}{c}0.82 \\
4 \\
\end{array}$ & 0.864 & 1.104 & 1.015 & 1.454 & 1.236 \\
\hline & SD & 0.197 & $\begin{array}{c}0.19 \\
6 \\
\end{array}$ & 0.197 & $\begin{array}{c}0.19 \\
1 \\
\end{array}$ & $\begin{array}{c}0.19 \\
3 \\
\end{array}$ & 0.187 & 0.097 & 0.024 & 0.614 & 0.800 \\
\hline & Median & 1 & 1 & 1 & 1 & $\begin{array}{c}0.83 \\
2\end{array}$ & 0.966 & 1.119 & 1.012 & 1.211 & 1.041 \\
\hline & $\mathrm{CV}$ & 0.226 & $\begin{array}{c}0.22 \\
5\end{array}$ & 0.226 & $\begin{array}{c}0.21 \\
8\end{array}$ & $\begin{array}{c}0.23 \\
4\end{array}$ & 0.216 & 0.088 & 0.023 & 0.422 & 0.647 \\
\hline & $\begin{array}{l}\text { No. } \\
\text { E>0.8/ } \\
\text { MPI>1 }\end{array}$ & 4 & 4 & 4 & 4 & 3 & 4 & 5 & 3 & 5 & 4 \\
\hline \multirow[b]{7}{*}{ 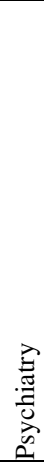 } & Max & 1 & 1 & 1 & 1 & 1 & 1 & 1.204 & 1.272 & 2.522 & 3.98 \\
\hline & Min & 0.577 & $\begin{array}{c}0.57 \\
7 \\
\end{array}$ & 0.577 & $\begin{array}{c}0.60 \\
9 \\
\end{array}$ & $\begin{array}{c}0.64 \\
4 \\
\end{array}$ & 0.605 & 1.020 & 0.965 & 0.840 & 0.842 \\
\hline & Mean & 0.727 & $\begin{array}{c}0.72 \\
5 \\
\end{array}$ & 0.720 & $\begin{array}{c}0.74 \\
1 \\
\end{array}$ & $\begin{array}{c}0.73 \\
9 \\
\end{array}$ & 0.734 & 1.053 & 1.035 & 1.079 & 1.143 \\
\hline & SD & 0.132 & $\begin{array}{c}0.12 \\
7 \\
\end{array}$ & 0.129 & $\begin{array}{c}0.13 \\
1 \\
\end{array}$ & $\begin{array}{c}0.11 \\
4 \\
\end{array}$ & 0.115 & 0.039 & 0.054 & 0.295 & 0.566 \\
\hline & Median & 0.683 & $\begin{array}{c}0.68 \\
7 \\
\end{array}$ & 0.675 & $\begin{array}{c}0.69 \\
9 \\
\end{array}$ & $\begin{array}{c}0.69 \\
6 \\
\end{array}$ & 0.700 & 1.05 & 1.031 & 1.033 & 1.031 \\
\hline & $\mathrm{CV}$ & 0.181 & $\begin{array}{c}0.17 \\
5 \\
\end{array}$ & 0.179 & $\begin{array}{c}0.17 \\
7 \\
\end{array}$ & $\begin{array}{c}0.15 \\
4 \\
\end{array}$ & 0.157 & 0.037 & 0.052 & 0.273 & 0.495 \\
\hline & $\begin{array}{ll}\text { No. } & \text { of } \\
\text { E }>0.8 & \\
\text { /MPI }>1 & \\
\end{array}$ & 6 & 5 & 6 & 6 & 4 & 7 & 26 & 22 & 19 & 18 \\
\hline \multirow{7}{*}{ 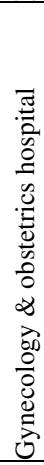 } & Max & 1 & 1 & 1 & 1 & 1 & 1 & 1.132 & 1.165 & 1.439 & 1.316 \\
\hline & Min & 0.578 & $\begin{array}{c}0.57 \\
2 \\
\end{array}$ & 0.574 & $\begin{array}{c}0.57 \\
7 \\
\end{array}$ & $\begin{array}{c}0.57 \\
9 \\
\end{array}$ & 0.580 & 0.905 & 0.915 & 0.685 & 0.357 \\
\hline & Mean & 0.700 & $\begin{array}{c}0.74 \\
1 \\
\end{array}$ & 0.744 & $\begin{array}{c}0.76 \\
6 \\
\end{array}$ & $\begin{array}{c}0.75 \\
1 \\
\end{array}$ & 0.740 & 1.022 & 1.015 & 1.058 & 0.931 \\
\hline & SD & 0.145 & $\begin{array}{c}0.17 \\
3 \\
\end{array}$ & 0.172 & $\begin{array}{c}0.17 \\
0 \\
\end{array}$ & $\begin{array}{c}0.16 \\
2 \\
\end{array}$ & 0.143 & 0.038 & 0.052 & 0.158 & 0.185 \\
\hline & Median & 0.636 & $\begin{array}{c}0.64 \\
2\end{array}$ & 0.645 & $\begin{array}{c}0.69 \\
0\end{array}$ & $\begin{array}{c}0.68 \\
6\end{array}$ & 0.684 & 1.02 & 1.019 & 1.025 & 0.953 \\
\hline & $\mathrm{CV}$ & 0.207 & $\begin{array}{c}0.23 \\
3 \\
\end{array}$ & 0.231 & $\begin{array}{c}0.22 \\
2 \\
\end{array}$ & $\begin{array}{c}0.21 \\
6 \\
\end{array}$ & 0.193 & 0.038 & 0.051 & 0.149 & 0.199 \\
\hline & $\begin{array}{ll}\text { No. } & \text { of } \\
E>0.8 / & \\
M P I>1 & \\
\end{array}$ & 5 & 7 & 8 & 8 & 7 & 9 & 23 & 15 & 17 & 9 \\
\hline \multirow{6}{*}{ 党 } & Max & 1 & 1 & 1 & 1 & 1 & 1 & 1.126 & 1.029 & 1.077 & 1.12 \\
\hline & Min & 0.619 & $\begin{array}{c}0.62 \\
0 \\
\end{array}$ & 0.621 & $\begin{array}{c}0.60 \\
8 \\
\end{array}$ & $\begin{array}{c}0.64 \\
8 \\
\end{array}$ & 0.623 & 1.011 & 0.764 & 0.876 & 0.859 \\
\hline & Mean & 0.777 & $\begin{array}{c}0.77 \\
6\end{array}$ & 0.747 & $\begin{array}{c}0.75 \\
1\end{array}$ & $\begin{array}{c}0.79 \\
4\end{array}$ & 0.769 & 1.053 & 0.969 & 0.988 & 0.976 \\
\hline & SD & 0.173 & $\begin{array}{c}0.17 \\
3 \\
\end{array}$ & 0.160 & $\begin{array}{c}0.15 \\
8 \\
\end{array}$ & $\begin{array}{c}0.15 \\
7 \\
\end{array}$ & 0.161 & 0.031 & 0.082 & 0.066 & 0.080 \\
\hline & Median & 0.660 & $\begin{array}{c}0.66 \\
0\end{array}$ & 0.651 & $\begin{array}{c}0.66 \\
8 \\
\end{array}$ & $\begin{array}{c}0.71 \\
2 \\
\end{array}$ & 0.672 & 1.049 & 0.988 & 1.003 & 0.977 \\
\hline & $\mathrm{CV}$ & 0.222 & $\begin{array}{c}0.22 \\
3 \\
\end{array}$ & 0.215 & $\begin{array}{c}0.21 \\
0 \\
\end{array}$ & $\begin{array}{c}0.19 \\
8 \\
\end{array}$ & 0.209 & 0.029 & 0.085 & 0.067 & 0.081 \\
\hline
\end{tabular}




\begin{tabular}{|c|c|c|c|c|c|c|c|c|c|c|c|}
\hline & $\begin{array}{l}\text { No. } \\
\text { E>0.8/ } \\
\text { MPI }>1\end{array}$ & 3 & 3 & 3 & 3 & 3 & 3 & 9 & 4 & 5 & 3 \\
\hline \multirow{7}{*}{ 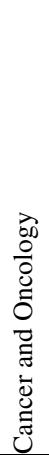 } & Max & 1 & 1 & 1 & 1 & 1 & 1 & 1.181 & 1.147 & 1.143 & 1.143 \\
\hline & Min & 0.664 & $\begin{array}{c}0.66 \\
8\end{array}$ & 0.648 & $\begin{array}{c}0.63 \\
6\end{array}$ & $\begin{array}{c}0.63 \\
6\end{array}$ & 0.663 & 0.926 & 0.950 & 0.530 & 0.794 \\
\hline & Mean & 0.877 & $\begin{array}{c}0.86 \\
2 \\
\end{array}$ & 0.799 & $\begin{array}{c}0.88 \\
6 \\
\end{array}$ & $\begin{array}{c}0.90 \\
4 \\
\end{array}$ & 0.866 & 1.053 & 1.045 & 0.964 & 0.975 \\
\hline & SD & 0.160 & $\begin{array}{c}0.14 \\
1 \\
\end{array}$ & 0.146 & $\begin{array}{c}0.15 \\
1 \\
\end{array}$ & $\begin{array}{c}0.14 \\
1 \\
\end{array}$ & 0.124 & 0.076 & 0.068 & 0.200 & 0.134 \\
\hline & Median & 1 & $\begin{array}{c}0.83 \\
9\end{array}$ & 0.781 & 1 & 1 & 0.858 & 1.049 & 1.029 & 1.031 & 0.984 \\
\hline & $\mathrm{CV}$ & 0.183 & $\begin{array}{c}0.16 \\
4\end{array}$ & 0.183 & $\begin{array}{c}0.17 \\
0\end{array}$ & $\begin{array}{c}0.15 \\
6\end{array}$ & 0.144 & 0.072 & 0.065 & 0.207 & 0.138 \\
\hline & $\begin{array}{l}\text { No. } \\
E>0.8 / \\
M P I>1\end{array}$ & 5 & 5 & 2 & 4 & 5 & 4 & 6 & 5 & 5 & 3 \\
\hline
\end{tabular}

95

96

\section{- Benchmarking}

97

98 There are two general ways to improve hospitals' productivity: reducing input

99 and/or increasing output. Table 7 presents the total inputs that need to be reduced

100 and the outputs that need to be increased to improve hospitals' efficiency in 2015.

101

102 Table 7. Total input reductions and output improvement needed to improve hospitals' efficiency.

\begin{tabular}{|c|c|c|c|c|c|c|c|c|c|}
\hline \multirow[b]{2}{*}{$\begin{array}{l}\text { Input/output } \\
\text { variables }\end{array}$} & \multicolumn{3}{|c|}{ General hospitals } & \multicolumn{3}{|c|}{ Specialized hospitals } & \multicolumn{3}{|c|}{ Psychiatry } \\
\hline & $\begin{array}{l}\text { Actual } \\
\text { values }\end{array}$ & $\begin{array}{l}\text { Target } \\
\text { values }\end{array}$ & $\begin{array}{c}\text { Differenc } \\
\text { e }\end{array}$ & $\begin{array}{l}\text { Actual } \\
\text { values }\end{array}$ & $\begin{array}{l}\text { Target } \\
\text { values }\end{array}$ & $\begin{array}{c}\text { Differenc } \\
\text { e }\end{array}$ & $\begin{array}{l}\text { Actual } \\
\text { values }\end{array}$ & $\begin{array}{l}\text { Target } \\
\text { values }\end{array}$ & Difference \\
\hline N. Physician & 20670 & 19552 & -1118 & 2997 & 2618 & -379 & 466 & 373 & -93 \\
\hline N. Nurse & 91,307 & 81,262 & -10045 & 14495 & 12090 & -2405 & 3055 & 2392 & -663 \\
\hline N. Other staff & 90456 & 82669.816 & -7786 & 16685 & 13424 & -3261 & 3707 & 2816 & -891 \\
\hline N. Hospital bed & 67145 & 66959 & -186 & 12858 & 9098 & -3760 & 6209 & 5935 & -274 \\
\hline N. Inpatient & 6483827 & 18782446 & 12298619 & 982771 & 1233193 & 250422 & 94523 & 143216 & 48693 \\
\hline N. Outpatient & 659592515 & 1472166350 & 812573835 & 71158752 & 72284878 & 1126126 & 39101261 & 46673032 & 7571771.5 \\
\hline $\begin{array}{l}\text { N. Surgical } \\
\text { operation }\end{array}$ & 2990767 & 7493631 & 4502864 & 467554 & 545734 & 78180 & 30931 & 44905 & 13974 \\
\hline $\begin{array}{l}\text { Average length of } \\
\text { stay }\end{array}$ & 3 & 3 & 0 & 6.8 & 3 & -3.8 & 24 & 37 & 13 \\
\hline $\begin{array}{ll}\text { Bed } & \text { occupation } \\
(\%) & \\
\end{array}$ & 68 & 85 & 17 & 73.5 & 855.2 & 781.7 & 84.6 & 98.7 & 14.1 \\
\hline $\begin{array}{l}\text { Number of bed } \\
\text { days }\end{array}$ & $12,253,963$ & $20,831,736.25$ & $8,577,774$ & 39104 & 378023 & 338919 & 59711 & 65562 & 5851 \\
\hline $\begin{array}{ll}\text { Degree } & \text { of } \\
\text { accreditation } & \end{array}$ & 3 & 4 & 1 & 3 & 4 & 1 & 3 & 4 & 1 \\
\hline
\end{tabular}

103

104

105

106

107

\section{Discussion}

108 
The objective of this study was to analyze the efficiency of government public hospitals, categorized by their specialty, over the period of 2012 to 2016 in Iran. Our findings revealed the overall low scores in the public hospitals' efficiency. All in all, public hospitals in Iran are not adequately efficient. For example, the average efficiency score in teaching hospitals and public hospitals is 0.488 and 0.357 , respectively, which is in line with the results of some other studies ${ }^{22,32}$. Only 7 hospitals had a score of above 0.8 per year. The MoHME's policy and social considerations to ensure the equitable geographical accessibility of hospitals nationwide, irrespective of their economic of scale, might be one of the reasons for low efficiency ${ }^{22}$. In a large country size as Iran, the challenge of improving hospitals' efficiency while ensuring geographical accessibility can be overcome through provision of high-quality clinical services, encouraging patients in need of elective services to use local services, rather than seeking care in provincial centers, and adjusting the bed number with the proportion of physicians and nurses accordingly, in line with the population size and the services they need.

It should be noted that a number of hospitals in this study were in the early years of their establishment. Newly established hospitals operate inefficiently in the early years due to shortcomings that they may experience at the outset of their activities. The MPI indicates progress in the average efficiency score of these hospitals over the period of analysis.

The average efficiency score of teaching hospitals varied between 0.354 and 1 . On average, only four out of the 130 teaching hospitals had scores above 0.8 . The overall low efficiency of these hospitals might be due to more input required for simultaneous provision of services and training. The efficiency scores in teaching and research hospitals were meaningfully higher than the other two groups of general and teaching hospitals. This could be due to the small difference between hospital inputs and outputs in this group and, conversely, a big difference between input and output variables in the other two hospital groups. Moreover, there are only a limited number of teaching and research hospitals across the country, with more or less bed numbers similar to other hospitals (less than 20 hospitals) that provide specialized, complex and unique services to a large number of patients.

The efficiency score of accident and burn hospitals in provincial centers and other regions was 1 and below 0.7, respectively. Most trauma and burn hospitals witnessed progress in their efficiency scores over the five years (MPI> 1). The highest and the lowest improvement were observed in 2012-2013 (MPI = 1.038) and 2015-2016 (MPI = 0.964), respectively. 
149 The efficiency score in pediatric hospitals, especially in the referral hospitals was

150 high. Adjusting the beds number and performance level of these hospitals in less 151 populated areas may contribute to improving their efficiency. Similar to other 152 specialized hospitals, the efficiency score of psychiatric hospitals was high, while 153 their range of variations was low.

\section{4}

The variations' range $(\mathrm{R})$ in specialized gynecology and obstetrics hospitals was higher than other specialized hospitals $(R=0.420)$, their efficiency score was low. This could be due to the low bed occupancy rate of these hospitals (Mean $<75 \%$ ), which might in turn be the result of the presence of similar wards in many public hospitals.

159 Logical reduction of these wards in general hospitals may enhance the efficiency of 160 these specialized hospitals.

161

162 Similar to other specialized hospitals, Cardiology specialized hospitals in the capital 163 city of Tehran had the efficiency score of 1 , whereas the score in similar hospitals 164 located in other cities was below 0.7. Again, this calls for the review of the existence 165 or absence of super-specialized hospitals across various geographical regions, aiming 166 to adjust their infrastructures with the existing demand and other social factors.

168 While the average efficiency score in cancer and oncology specialized hospitals was $1690.865(\mathrm{SD}=0.12)$, it ranged between 0.662 and $1(\mathrm{R}=0.337)$ over the analysis years. On 170 average, four out of seven hospitals in this group had scores of above 0.8 .

171 In Iran Educational hospitals are less efficient than None-educational hospitals and 172 that specialized hospitals are also the most efficient. The fact that they are targeting 173 different patients and may have different technologies does not change the 174 conclusion because the efficiency is just measuring how input mobilization translates 175 in out puts. So the study demonstrates that productivity of workforce and beds are 176 higher in the specialized hospitals than in other categories. Also, the issue of limited 177 efficient of Educational hospitals can be related to the measurement of staff as part of 178 their time can be dedicated to education, then this could be also better captured in 179 the information system in place with an approach based on the Full Time Equivalent 180 (FTE) measure of staff rather than head count i.e. a doctor dedicated 50\% of time for 181 education is considered as 0.5 FTE for clinical activities, etc...

182 Based on findings, over time the efficiency remains within a category and this is an 183 expected result because changes would take longer period than 4 years to be visible. 184 From previous DEA work efficiency is compared over a period of over 10 years. This 185 is confirming that health policies cannot have short term impact on hospital 186 efficiency. 
A recent study calculated and analysed the efficiency of all public hospitals in Spain in $2017^{18}$ and reported the average efficiency score 0.736 , very similar to our revealed score of 0.732 . The study compared similar hospitals with each other. In our study, we used more output variables to enhance the reliability of analysis. A systematic review showed that $90 \%$ of studies used the DEA method to measure the efficiency of hospitals in Iran, and the calculated score ranged from 0.7 to 0.933 . A similar study in China also used DEA method ${ }^{34}$. They used the number of beds as the input variable and the hospitalization days, the number of visits, and the number of surgical operations as the most used output variables for measuring the efficiency. A Chinese study measured the efficiency of government hospitals to examine the impact of the country-wide development plan of 2009 on the efficiency of a sample of 114 hospitals. They used similar input and output variables to our study and calculated the average efficiency score of 0.748 , while significant potential for improving the technical efficiency of the hospitals was reported ${ }^{35}$.

Another study used similar input and output indicators to examine the efficiency of health services centers in Indonesia. They used Pabón-Lasso model. Forty percent of hospitals and 33 percent of health centers were located in the high performing sector of the Pabón-Lasso model.

${ }^{36}$. Another study used 10 variables to measure the efficiency of Turkish hospitals in 2015 and found that only $17 \%$ of the total 1103 hospitals were efficient ${ }^{37}$. A similar study in Turkey that examined the efficiency of 1079 hospitals reported that the government hospitals affiliated with the Turkish Ministry of Health were more efficient than the private hospitals ${ }^{38}$. A study conducted in Greece to assess the impact of sanctions on the efficiency of hospitals between 2009 and 2012 found that the rate of efficiency declined over the mentioned years (MPI $=0.72)^{39}$. The MPI in our study was 1.07 .

214 In the end, it should be noted that the efficiency score over the years under review 215 was not significantly altered, and most of the changes were related to Orthopaedics and Accidents and Burns hospitals.

\section{Rigor of study}

This study began to use the Extended-DEA method as the most utilized technique to measure hospitals' efficiency worldwide. Our findings were hard to interpret, so we engaged with a scholarly team in applied mathematics to revise the method and invented Extended-DEA as a result, which is the main strength of our study. In addition, we included all public hospitals in the country and categorized them into various specialties. Comparing similar DMUs is one of the basic assumptions to 
measure efficiency ${ }^{40}$, so we classified hospitals in similar groups. We also brought 227 some qualitative variable as output indicators (Accreditation Degree of hospitals) in our calculation, which is usually neglected in efficiency measurement studies. Last but not least, although most similar studies measured efficiency in a cross-sectional study design, we measured the trend of efficiency in all categories of public hospitals over a period of five years (2012-2016).

Limitations

233 Despite advantages, our study had some limitations. Due to shortages in an established monitoring system to collect the related data on hospital efficiency, reliable and valid hospital data with enough input and output variables is not available in Iran. Further, despite our efforts to obtain data form the MoHME, which is the most reliable and available sources in Iran, there still remains some limits in data credibility that might affect the reliability of our data source. Nevertheless, there is no other source of data available to conduct analysis as in this study.

240 Our study could have benefited from some technical considerations to enhance accuracy of the findings. We advocate more robust approach in designing studies on the efficiency of hospitals. In particular, use of the international standards in selection of input and output indicators and paying enough attention to homogenization of the unit of indicators (monetary, volumetric, relative, etc.,) are important. Moreover, it is crucial to bring the number of DMUs three times higher than the input and output indicators, as well as combine the current two-stage and three-stage DEA models with the Bootstrap-DEA method to calculate more accuracy efficiency ${ }^{41}$.

\section{Policy implications}

- Identifying the current status of hospital efficacy is the first step to improve productivity. Executive managers and policy makers can use our findings as reliable evidence for better and more efficient use of resources with the aim of improving productivity.

- Given the country-wide need to employ more healthcare workers in hospitals, decision makers can build on our results that show the possible output expansions and/or the possible input contractions and prioritize efforts for improvements. For instance, hospitals with a surplus capacity of input variables (including health workers and bed), may decide to reduce their additional inputs during a comprehensive planning process.

- To address the lower efficiency of specialized hospitals in some provinces, we recommend redistribution of the specialized beds and other resources in some target regions. 
- The integration of small hospitals in some areas can help improve productivity, taking into account the criteria for access to services.

- The hospital efficiency measurement is only valid for homogenous category of hospitals. This is well demonstrated in our fig 1 and is the most important message from future studies.

\section{Conclusions}

We measured the efficiency scores and MPI for 568 public hospitals during 20122016, using an Extended-DEA for the first time in Iran. Given the overall low efficiency scores of hospitals, especially general hospitals that are not adequately efficient or are merely inefficient, within various specialties across the country, robust and evidence-based measures are required for more efficient use of resources. As Iran has been implementing its ambitious health transformation plan (HTP) to reach universal health coverage by 2025 , and considering the historical shortages in hospital particularly in deprived areas as well as the financial limitations to build new hospitals, integrating local general clinics and other hospitals in small communities could be a reasonable policy to enhance the efficiency of the existing resources. Unless hospital managers in Iran improve their ability for need-based allocation of human resources among various settings, enhance the quality of hospital services to increase their revenue and stabilize their financial status, e.g. through the use of prospective payment systems, and manage to optimize utilization of the physical resources, hospital care cannot cope with the ever-increasing demand and the health system may suffer from the upcoming challenges even further.

\section{Declarations}

Ethics approval and consent to participate: This study was approved by the ethical

290 IR.TUMS.VCR.REC.1396.4018.

291 Consent for publication: Not applicable.

292 Availability of data and materials: The data of this study are available in

293 "Supplementary Material"

294 Competing interests: None of the authors have any competing interests.

295 Funding: This study founded by the MoHME's Iran

296 Authors' contributions: AT, AO and RM conceived the study and designed its method. EM performed the computations and applied the model, with help from

298 FHL for revision of the analytical method. All authors discussed the results and contributed to the final manuscript. EM HSH and HY carried out the analytical

300 experiment. EM and AHT wrote the manuscript all authors contributed to the 301 development and approved the final manuscript. AT is the guarantor. 

hospital data. Also, The authors thank Dr. Eric de Roodenbeke for critical reviewing

304 of the manuscript

\section{References}

306 1. Manyazewal T. Using the World Health Organization health system building blocks through survey of healthcare professionals to determine the performance of public healthcare facilities. Archives of Public Health. 2017;75(1):50.

2. Pourreza A, Alipour V, Arabloo J, Bayati M, Ahadinezhad B. Health production and determinants of health systems performance in WHO Eastern Mediterranean Region. Eastern Mediterranean Health Journal. 2017;23(5).

3. Raei B, Yousefi M, Rahmani K, Afshari S, Ameri H. Patterns of productivity changes in hospitals by using Malmquist-DEA Index: A panel data analysis (2011-2016). Australasian Medical Journal (Online). 2017;10(10):856-864.

4. Kiani MM, Raei B, Asbagharani PA, et al. Comparison of the technical efficiency of the hospital sector: Panel data analysis of the Iranian hospitals using parametric and nonparametric approaches.

5. Minges KE, Curtis J, Desai N, et al. PERFORMANCE AND CHARACTERISTICS OF HOSPITALS PARTICIPATING IN THE AMERICAN COLLEGE OF CARDIOLOGY VOLUNTARY PUBLIC REPORTING PROGRAM. Journal of the American College of Cardiology. 2018;71(11):A498.

6. Navathe AS, Liao JM, Shah Y, et al. Characteristics of hospitals earning savings in the first year of mandatory bundled payment for hip and knee surgery. Jama. 2018;319(9):930-932.

7. Division UP, Economic Do, Staff SAPD. Population, Development and HIV/AIDS with Particular Emphasis on Poverty: The Concise Report. Vol 247: United Nations Publications; 2005.

8. Poortinga W, Grey C, Jiang S, et al. Short-term health and social impacts of energy-efficiency investments in low-income communities: a controlled field study. The Lancet. 2016;388:S96.

9. Mobinizadeh M, Raeissi P, Nasiripour AA, Olyaeemanesh A, Tabibi SJ. A model for priority setting of health technology assessment: the experience of AHP-TOPSIS combination approach. DARU Journal of Pharmaceutical Sciences. 2016;24(1):10.

10. Gannon B. Testing for variation in technical efficiency of hospitals in Ireland. Vol. XX, No. XX, Issue, Year. 2005.

11. Barros CP, Athanassiou M. Efficiency in European seaports with DEA: evidence from Greece and Portugal. Port Management: Springer; 2015:293-313.

12. Ersoy K, Kavuncubasi S, Ozcan YA, Harris II JM. Technical efficiencies of Turkish hospitals: DEA approach. Journal of Medical Systems. 1997;21(2):67-74.

13. Kirigia JM, Emrouznejad A, Sambo LG. Measurement of technical efficiency of public hospitals in Kenya: using data envelopment analysis. Journal of Medical Systems. 2002;26(1):39-45.

14. Ramanathan R. Operations assessment of hospitals in the Sultanate of Oman. International Journal of Operations \& Production Management. 2005;25(1):39-54.

15. Ghaderi H, Godarzi A, Gohary M. Determining Of Technical Efficiency In Iran University Of Medical Science's Hospital By Using DEA Method: 2000-2004. Journal of Health Administration. 2006;9(26):31-38.

16. MOHAMMADI AM, MIRGHAFOURI S, MIRFAKHR AS, DAMAKI A, MOUMENI H. EVALUATION OF THE RELATIVE EFFICIENCY OF GOVERNMENT HOSPITALS IN YAZD USING DEA MODEL (DATA ENVELOPMENT ANALYSIS). 2009.

17. Azad E, Ketabi S, Soltani I, Bagherzade M. Analysis of efficiency and resource allocation at different wards in Shariati hospital, Isfahan, Iran, using data envelopment analysis. Health Information Management. 2012;8(7):938-947.

18. Perez-Romero C, Ortega-Díaz M, Ocana-Riola R, Martín-Martín J. Analysis of the technical efficiency of hospitals in the Spanish National Health Service. Gaceta sanitaria. 2017;31(2):108-115.

19. Dong S, Zuo Y, Guo S, Li M, Liu X, Li H. Data envelopment analysis for relative efficiency measurements of Chinese hospitals: a systematic review. Research Heal Sci. 2017;2(2):79103. 
20. Pelone F, Kringos DS, Romaniello A, Archibugi M, Salsiri C, Ricciardi W. Primary care efficiency measurement using data envelopment analysis: a systematic review. Journal of Medical Systems. 2015;39(1):156.

\section{review. 2016.}

21. Katharakisa G, Katostaras T. SFA vs. DEA for measuring healthcare efficiency: A systematic

22. Mosadeghrad AM, Esfahani P, Nikafshar M. Hospitals' efficiency in Iran: A systematic review and meta-analysis of two decades of research. Journal of Payavard Salamat. 2017;11(3):318-331.

23. Khangah HA, Jannati A, Imani A, Salimlar S, Derakhshani N, Raef B. Comparing the health care system of Iran with various countries. Health Scope. 2017;6(1).

24. Delgoshaei B, Mobinizadeh M, Mojdekar R, Afzal E, Arabloo J, Mohamadi E. Telemedicine: A systematic review of economic evaluations. Medical journal of the Islamic Republic of Iran. 2017;31:113.

25. Varabyova Y, Müller J-M. The efficiency of health care production in OECD countries: a systematic review and meta-analysis of cross-country comparisons. Health Policy. 2016;120(3):252-263.

26. Najafpour Z, Hasoumi M, Behzadi F, Mohamadi E, Jafary M, Saeedi M. Preventing blood transfusion failures: FMEA, an effective assessment method. BMC health services research. 2017;17(1):453.

27. Mohamadi E, Tabatabaei SM, Olyaeemanesh A, et al. Coverage Decision-Making for Orthopedics Interventions in the Health Transformation Program in Iran: A Multiple Criteria Decision Analysis (MCDA). Shiraz E-Medical Journal. 2016;17(12).

28. Kalhor R, Salehi A, Keshavarz A, Bastani P, Heidari Orojloo P. Assessing hospital performance in Iran using the Pabon Lasso Model. Asia Pacific Journal of Health Management. 2014.

29. Rezapour A, Foroughi Z, Sadeghi NS, et al. Identification of the most appropriate variables for measuring the efficiency of Iranian public hospitals: Using Delphi technique. Journal of Education and Health Promotion. 2019;8.

30. Giancotti M, Mauro M. Scale Efficiency of Public Hospital: A Content Analysis of 20 Years of Research. World Review of Business Research. 2015;5(2):24-38.

31. Du J, Chen Y, Huang Y. A modified Malmquist-luenberger productivity index: Assessing environmental productivity performance in China. European Journal of Operational Research. 2018;269(1):171-187.

32. Bahadori M, Izadi AR, Ghardashi F, Ravangard R, Hosseini SM. The evaluation of hospital performance in Iran: a systematic review article. Iranian journal of public health. 2016;45(7):855.

33. Kiadaliri AA, Jafari M, Gerdtham U-G. Frontier-based techniques in measuring hospital efficiency in Iran: a systematic review and meta-regression analysis. BMC health services research. 2013;13(1):312.

34. Dong S, Zuo Y, Guo S, Li M, Liu X, Li H. Data Envelopment Analysis for Relative Efficiency Measurement of Chinese Hospitals: A Systematic Review. Research in Health Science. 2017;2(2):79.

35. Cheng Z, Tao H, Cai M, et al. Technical efficiency and productivity of Chinese county hospitals: an exploratory study in Henan province, China. BMJ open. 2015;5(9):e007267.

36. Hafidz F, Ensor T, Tubeuf S. Assessing health facility performance in Indonesia using the Pabón-Lasso model and unit cost analysis of health services. The International journal of health planning and management. 2018.

37. Narc1 HÖ, Ozcan YA, Şahin İ, Tarcan M, Narc1 M. An examination of competition and efficiency for hospital industry in Turkey. Health care management science. 2015;18(4):407418.

38. Yildiz MS, Heboyan V, Khan MM. Estimating technical efficiency of Turkish hospitals: implications for hospital reform initiatives. BMC health services research. 2018;18(1):401.

39. Xenos P, Yfantopoulos J, Nektarios M, Polyzos N, Tinios P, Constantopoulos A. Efficiency and productivity assessment of public hospitals in Greece during the crisis period 2009-2012. Cost Effectiveness and Resource Allocation. 2017;15(1):6.

40. Jacobs R, Smith PC, Street A. Measuring efficiency in health care: analytic techniques and health policy: Cambridge University Press; 2006.

41. Tlotlego N, Nonvignon J, Sambo LG, Asbu EZ, Kirigia JM. Assessment of productivity of hospitals in Botswana: a DEA application. International archives of medicine. 2010;3(1):27. 
417

418 


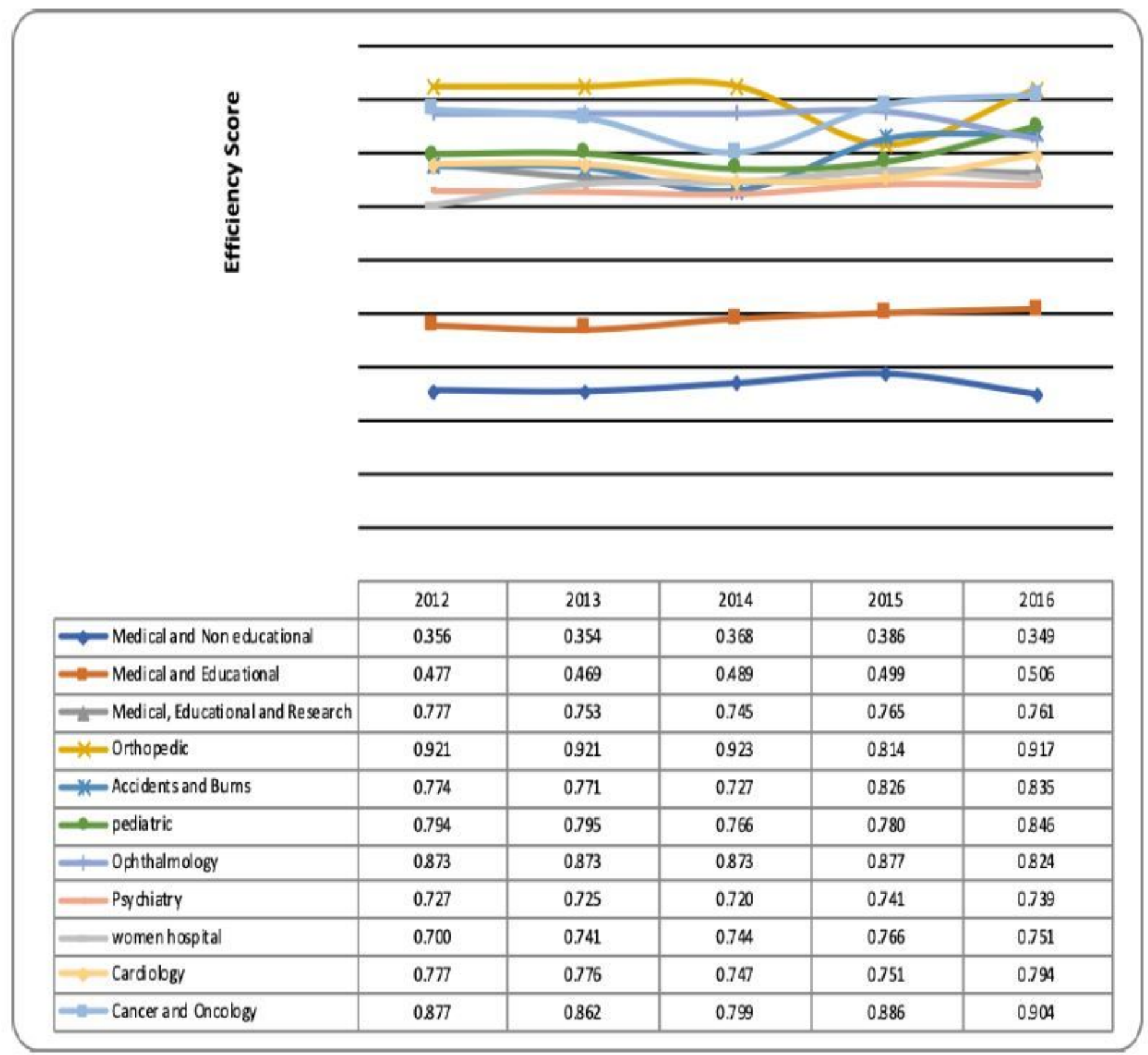

Figure 1

Average efficiency score by hospital type, 2012-16.

\section{Supplementary Files}

This is a list of supplementary files associated with this preprint. Click to download.

- Appendixes.docx 
- SupplementaryMaterial.rar 